\title{
A child with painless left wrist swelling
}

\section{Wai Keung Lo ${ }^{1}$ (D) $\cdot$ Cheuk Kei Kathy Wong ${ }^{1} \cdot$ Chi Hin Chan ${ }^{1}$}

Published online: 11 January 2018

(C) ISS 2018

A 9-year-old child presented with insidious onset painless left wrist swelling for months. (Figs. 1, 2, 3, 4, 5, and 6).

Fig. 1 Radiographs of left wrist

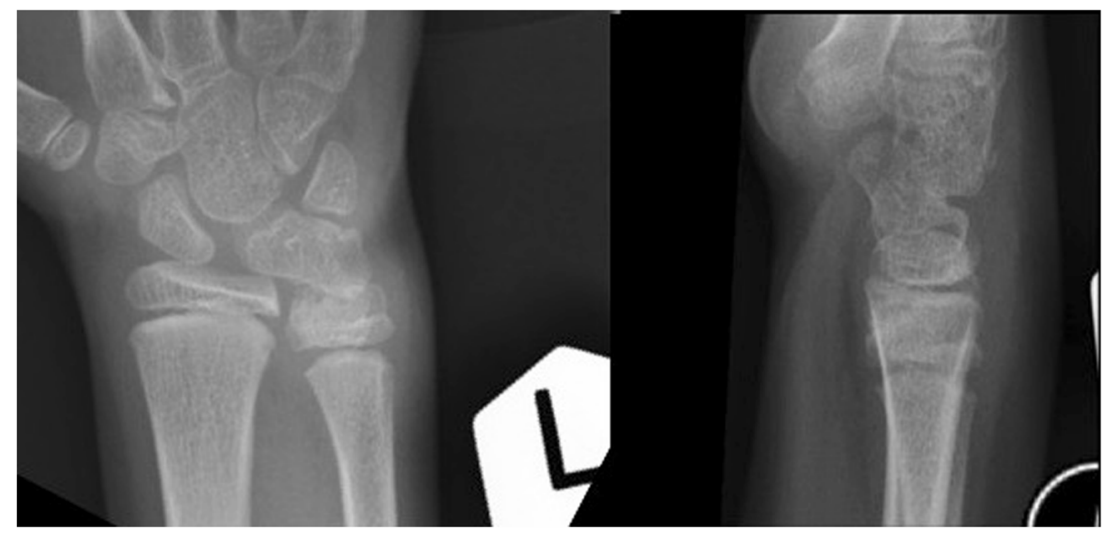

Fig. 2 Coronal CT of left wrist

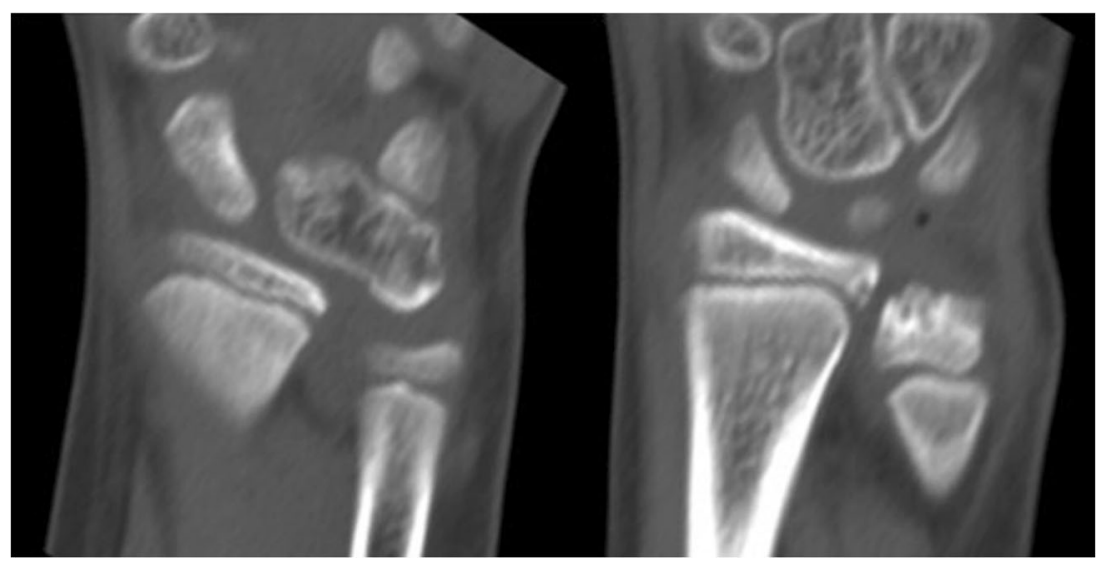

The diagnosis can be found at https://doi.org/10.1007/s00256-017-2859-3

Wai Keung Lo

waikglo@gmail.com

1 Department of Diagnostic and Interventional Radiology, Kwong

Wah Hospital, 25 Waterloo Road, Yau Ma Tei, Hong Kong 
Fig. 3 Sagittal CT at sites of pathology

Fig. 4 3D-reformatted CT showing volar, ulnar and dorsal sides of wrist

Fig. 5 Coronal T1W MRI of left wrist

Fig. 6 Coronal water-selective sequence highlighting cartilages of left wrist
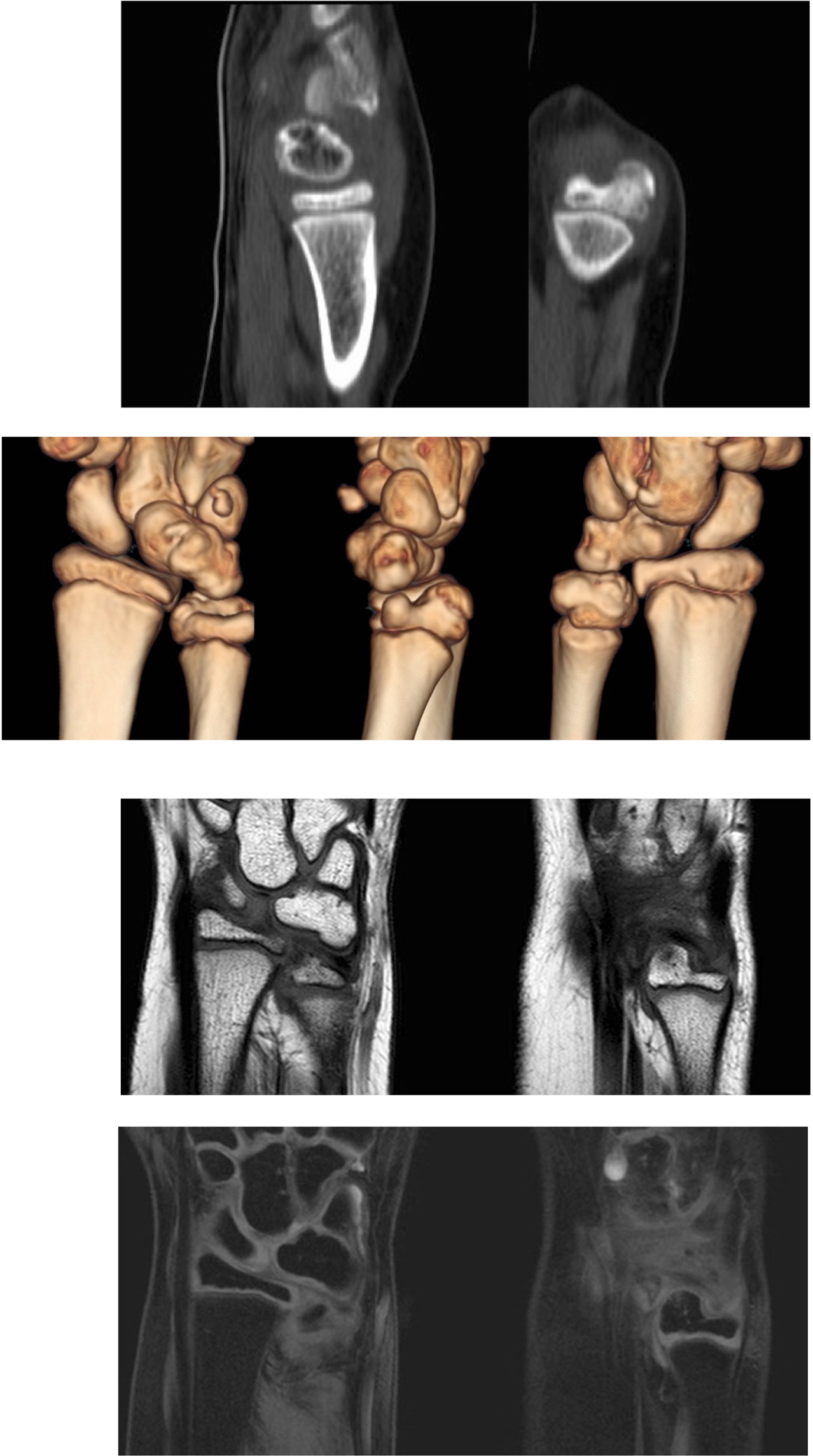